\title{
Transição nutricional no Brasil: análise dos principais fatores
}

\section{Nutritional transition in Brazil: Analysis of the main factors}

\section{Elton Bicalho de Souza ${ }^{1}$}

\section{Palavras-chave:}

Transição nutricional

Obesidade

Nutrição

Atividade física

\section{Resumo}

É incontestável que o Brasil experimenta, nos últimos, uma rápida transição nutricional. Chama a atenção, o marcante aumento na prevalência de obesidade, consolidando-se como o agravo nutricional mais importante, sendo associado a uma alta incidência de doenças crônicas não transmissíveis, como diabetes e doenças cardiovasculares. Alterações no estilo de vida, como a má-alimentação e redução no gasto calórico diário são os principais fatores que explicam o crescimento da obesidade. $\mathrm{O}$ aumento da prevalência de sobrepeso e obesidade no Brasil é relevante, e o quadro epidemiológico nutricional necessita de estratégias de saúde pública, capazes de modificar padrões de comportamento alimentar e da atividade física. A presente revisão de literatura tem como objetivo avaliar os principais componentes da transição nutricional ocorrida no Brasil e suas consequências na população.

\section{Abstract}

It is undeniable that in the last years Brazil has undergone a fast nutritional transition. It calls the attention the increase of obesity held as the most important nutritional worsening, and associated to a high incidence of non-communicable chronic diseases, such as diabetes and cardiovascular diseases. Lifestyle changes, like poor eating and reduced energy expenditure are the main contributing factors to the increasing prevalence of obesity. The increase of overweight and obesity in Brazil is considerable, and nutritional epidemiological picture needs public health strategies able to change nutrition and physical activity patterns. The aim of this literature review is to evaluate the principal components of nutritional transition in Brazil and its consequences to the population.
Key words:

Nutritional transition

Obesity

Nutrition

Physical activity 


\section{Introdução}

Apesar de um cenário conturbado, envolvendo momentos marcantes nos campos da política, do esporte, da saúde e da educação; o Brasil, assim como outros países menos desenvolvidos, passou por importantes transformações no processo de saúde/doença. Principalmente nos últimos cinquenta anos, são observadas alterações na qualidade e na quantidade da dieta, e, associadas a mudanças no estilo de vida, nas condições econômicas, sociais e demográficas, observam-se repercussões negativas na saúde populacional desses países (BATISTA FILHO \& RISSIN, 2003; KAC \& VELÁSQUEZ-MELÉNDEZ, 2003). Como consequência desses fatos, a prevalência de sobrepeso e da obesidade aumenta consideravelmente e, consequentemente, as doenças crônicas não transmissíveis, principalmente diabetes, hipertensão arterial, doenças cardiovasculares e cânceres, acarretando em mudanças no padrão da distribuição das morbimortalidade das populações (SARTORELLI \& FRANCO, 2003). O objetivo desta revisão é realizar uma avaliação dos principais componentes da transição nutricional ocorrida no Brasil e suas consequências na população.

\section{Mudanças no padrão alimentar do brasileiro}

Apesar da atual visibilidade no cenário mundial - em função do desenvolvimento cultural, científico e econômico - o Brasil situa-se entre os países de maior prevalência em deficiências nutricionais (ESCODA, 2002).

Alterações nos padrões dietéticos e nutricionais da população brasileira de todos os estratos sociais e faixas-etárias vêm sendo analisadas no processo da transição nutricional. Essa transição nutricional caracteriza-se pela redução nas prevalências dos déficits nutricionais e aumento expressivo de sobrepeso e obesidade (BATISTA FILHO \& RISSIN, 2003).

Alguns aspectos tentam explicar este fenômeno: com relação a termos de ocupação demográfica, passamos de uma população rural (66\% nos anos 50) para uma condição de país predominantemente urbano $(80 \%$ das pessoas atualmente radicadas nos centros ur- banos); outro episódio importante é o fato da inserção da mulher no mercado de trabalho, principalmente na década de 70 , ocasionando uma família economicamente dependente da participação da renda da mulher no sustento da família. Com isso, perde-se um pouco da figura da mulher "dona do lar", o que transforma a qualidade da alimentação, uma vez que a mesma não dispõe de tanto tempo para preparar as refeições com tanta qualidade, preferindo alimentos industrializados, e até mesmo a realização das refeições fora do lar, principalmente em restaurantes, pensões e fast-foods (BATISTA FILHO \& RISSIN, 2003; KAC \& VELÁSQUEZ-MELÉNDEZ, 2003; MENDONÇA \& ANJOS, 2004).

Como consequência, observou-se um aumento do consumo de ácidos graxos saturados, açúcares, refrigerantes, álcool, produtos industrializados com excesso de ácidos graxos "trans", carnes, leite e derivados ricos em gorduras, guloseimas como doces, chocolates, balas, etc. Em contrapartida, foi constatada uma redução considerável no consumo de carboidratos complexos, frutas, verduras e legumes. Esse fato gera um quadro de excesso calórico por conta da elevada ingestão de macronutrientes (carboidratos, proteínas e lipídeos), e deficiência de micronutrientes (vitaminas e minerais) (ESCODA, 2002).

\section{Redução da prática de atividade física}

Concomitante com a alteração da característica da dieta, observa-se uma acentuada redução do nível de atividade física. Vários fatores podem explicar este fato, mas as principais mudanças estão na distribuição das ocupações setoriais (da agricultura para a indústria) e um aumento do número de trabalhos com redução do esforço físico ocupacional, em virtude do aumento da tecnologia (MENDONÇA \& ANJOS, 2004).

Também são observadas modificações nas atividades de lazer, que passam de atividades com gasto energético acentuado, como práticas esportivas e longas caminhadas, para atividades sedentárias, como televisão, videogame ou computador. Com relação às horas assistindo televisão, vale ressaltar dois prin- 
cipais aspectos: a diminuição do gasto energético, devido à redução da atividade física propriamente dita, e o aumento da ingestão de alimentos com alta concentração calórica em frente à televisão e/ou decorrente dos anúncios por ela veiculados. Segundo FRUTUOSO et al (2003), foi observado que os anúncios da TV norte-americana relacionados à alimentação transmitidos entre 20 e 23 horas, 60\% referiam-se a refrigerantes e outros produtos alimentícios açucarados, considerados perigosos quando consumidos em excesso.

A utilização de recursos tecnológicos também contribui efetivamente para o aumento do sedentarismo da população. Portões eletrônicos, escadas rolantes, vidros automáticos, veículos motorizados e o uso crescente de equipamentos domésticos como máquinas de lavar roupa e louça, ao invés de fazê-lo manualmente, são apontados como grandes responsáveis pela redução do gasto energético com atividades diárias (PITANGA, 2002; FRUTUOSO et al, 2003; MENDONÇA \& ANJOS, 2004).

\section{Aumento do sobrepeso e da obesidade}

Em resposta às modificações no padrão de comportamento alimentar e da drástica redução na prática de atividades físicas, a transição nutricional trouxe mudanças do perfil de saúde da população brasileira, sendo que o aumento das prevalências do sobrepeso e da obesidade são os principais legados desta transição (GiGANTE et al, 1997; MARINHO et al, 2003; PINHEIRO et al, 2004; COUTINHO, 2008).

A obesidade pode ser definida como o acúmulo excessivo de gordura corporal. Apesar das limitações de não considerar a diferença entre "massa magra" e "massa gorda", o Índice de Massa Corporal (IMC) é atualmente a ferramenta mais utilizada em estudos populacionais para o diagnóstico do excesso de gordura corporal, que é considerada um problema de Saúde Pública e, apesar de ainda relativamente incomum em países da África e da Ásia, pode ser considerada a mais importante desordem nutricional no mundo, por ter um aumento elevado de sua incidência. Estima-se que $10 \%$ da população dos países desenvolvidos tenham excesso de peso e mais de um terço dos norte-americanos estejam acima do peso (GUIMARÃES \& BARROS, 2001; COUTINHO, 2008).

No Brasil, segundo o Estudo Nacional da Despesa Familiar (ENDEF-1975) e a Pesquisa Nacional sobre Saúde e Nutrição (PNSN, 1989), no intervalo de tempo entre os dois estudos houve um aumento de sobrepeso na proporção de $58 \%$ para homens e $42 \%$ para mulheres, e mais alarmante foi o aumento da obesidade, registrando o incrível aumento de $100 \%$ para o sexo masculino e $70 \%$ para o sexo feminino. Atualmente, o excesso de peso afeta $40 \%$ da população, sendo que, desse grupo, a obesidade atinge $11,1 \%$ (FRANCISCHI et al, 2000; KAC \& VELÁSQUEZ-MELÉNDEZ, 2003; FERREIRA \& MAGALHÃES, 2006; NUNES et al, 2007; SARTURI et al, 2010).

As consequências do aumento do sobrepeso e da obesidade têm sido catastróficas. $\mathrm{O}$ excesso de peso é um dos principais fatores de risco para hipertensão arterial, hipercolesterolemia, diabetes mellitus, doenças cardiovasculares e algumas formas de câncer. Segundo a Organização Mundial da Saúde (OMS), a obesidade e a hipertensão arterial são os dois principais responsáveis pela maioria das mortes e doenças em todo mundo. Já no Brasil, as doenças cardiovasculares estão nas estatísticas de saúde como a primeira causa de morte há pelo menos quatro décadas. Nos últimos 20 anos, um aumento significativo na mortalidade por diabetes e algumas neoplasias malignas decorrentes de um estilo de vida ruim também merece atenção especial (FELIPPE \& SANTOS, 2004; FERREIRA \& MAGALHÃES, 2006).

Segundo o Consenso Latino Americano de Obesidade, cerca de 200 mil pessoas morrem por ano devido a doenças associadas ao excesso de peso e estima-se que nos Estados Unidos esse número seja de aproximadamente 300 mil pessoas. Estima-se que os gastos públicos com o excesso de peso, nos países desenvolvidos, seja de $2 \%$ a $7 \%$ dos orçamentos de saúde. No Brasil, este custo é de quase R \$ 1 bilhão por ano (SOUZA et al, 2003; FELIPPE \& SANTOS, 2004). 


\section{Considerações finais}

A comprovação de que a transição nutricional acarretou sérias alterações ao longo do tempo é notória. A diminuição progressiva da desnutrição e o aumento do excesso de peso, independente de idade, sexo ou classe social é uma realidade que há tempos os estudos demonstram.

Ao constatar tal evidência, faz-se necessária a mobilização das autoridades para a determinação de prioridades para a definição de estratégias de ação de Saúde Pública. Para o combate do excesso de peso e das doenças crônicas não transmissíveis, ações de educação alimentar e incentivo à prática de atividades físicas diárias merecem destaque.

Em virtude desses fatos, o Ministério da saúde publicou os dez passos para o peso saudável. Esse material é oriundo do Plano Nacional para a Promoção da Alimentação Adequada e do Peso Saudável, visando à modificação do estilo de vida da população brasileira, principalmente, sobre a prática alimentar e atividade física. Os dez passos são:

1. Comer frutas e verduras variadas, pelo menos duas vezes por dia;

2. Consumir feijão pelo menos quatro vezes por semana;

3. Evitar alimentos gordurosos como carnes gordas, salgadinhos e frituras;

4. Retirar a gordura aparente das carnes e a pele do frango;

5. Nunca pular refeições: fazer três refeições e um lanche por dia. No lanche escolher uma fruta;

6. Evitar refrigerantes e salgadinhos de pacote;

7. Fazer as refeições com calma e nunca na frente da televisão;

8. Aumentar a sua atividade física diária. Ser ativo é se movimentar. Evitar ficar parado, você pode fazer isto em qualquer lugar;

9. Subir escadas ao invés de usar o elevador, caminhar sempre que possível e não passar longos períodos sentado assistindo à $\mathrm{TV}$;

10. Acumular trinta minutos de atividade física todos os dias.
Essas atitudes já contribuem bastante para evitar um quadro e excesso de peso. Porém, a divulgação dos dez passos por si só não é satisfatória nem eficaz, e é cada vez mais notória a necessidade de políticas públicas mais eficientes, como projetos e programas intersetoriais objetivando a conscientização da necessidade de um estilo de vida mais ativo e de uma alimentação mais saudável. A médio e longo prazo, essas políticas reduziriam consideravelmente os gastos com o tratamento da obesidade e doenças subsequentes. Logo, recomenda-se uma atenção especial e medidas emergências para o combate a essa epidemia, evitando a proliferação das complicações decorrentes do excesso de peso, visando à preservação da vida de milhares de brasileiros.

Divulgação de campanhas educativas em rede nacional, incentivando o consumo de alimentos saudáveis e adoção de educação alimentar nas escolas, aumento do número de aulas de Educação Física e incentivos a prática esportiva são alternativas que devem ser adotadas imediatamente para tentar reverter o atual quadro da epidemia do excesso de peso.

\section{Referências bibliográficas}

1. BATISTA FILHO, M.; RISSIN, A. A transição nutricional no Brasil: tendências regionais e temporais. Cad. Saúde Pública, 19(Sup. 1): S181-S191, 2003.

2. GUIMARÃES, L.V.; BARROS, M.B.A. As diferenças de estado nutricional em pré-escolares de rede pública e a transição nutricional. J Pediatria, 77(5): 381-6, 2001.

3. KAC,G.;VELÁSQUEZ-MELÉNDEZ,G. A transição nutricional e a epidemiologia da obesidade na América Latina. Cad. Saúde Pública, 19(Sup. 1):S4-S5, 2003.

4. SARTORELLI, D.S.; FRANCO, L.J. Tendências do diabetes mellitus no Brasil: o papel da transição nutricional. Cad. Saúde Pública, 19 (Sup. 1): S29-S36, 2003.

5. ESCODA, M.S.Q. Para a crítica da transição nutricional Ciência \& Saúde Coletiva, 7(2): 219-226, 2002. 
6. MENDONÇA, C.P.; ANJOS, L.A. Aspectos das práticas alimentares e da atividade física como determinantes do crescimento do sobrepeso/obesidade no Brasil. Cad. Saúde Pública, 20(3): 698709, 2004.

7. PITANGA, F.J.G. Epidemiologia, atividade física e saúde. Rev. Bras. Ciên. e Mov., 10(3): 49-54, 2002.

8. FRUTUOSO, M.F.P. et al. Redução do dispêndio energético e excesso de peso corporal em adolescentes. Rev. Nutr., 16(3): 257-263, 2003.

9. PINHEIRO, A.R.O. et al. Uma abordagem epidemiológica da obesidade. Rev. Nutr., 17(4): 523-533, 2004.

10. MARINHO, SP et al. Obesidade em adultos de segmentos pauperizados da sociedade. Rev. Nutr., 16(2):195-201, 2003.

11. GIGANTE, D.P. et al. Prevalência de obesidade em adultos e seus fatores de risco. Rev. Saúde Pública, 31(3): 236246, 1997.

12. FRANCISCHI, R.P.P. et al. Obesidade: etiologia, morbidade e tratamento. Rev. Nutr., 13(1): 17-28, 2000.

13. COUTINHO, J.G. et al. A desnutrição e obesidade no Brasil: o enfrentamento com base na agenda única da nutrição. Cad. Saúde Pública, 24(Sup 2):S332S340, 2008.

14. SOUZA, L.J. et al. Prevalência de obesidade e fatores de risco cardiovascular em Campos, Rio de Janeiro. Arq Bras Endocrinol Metab., 47(6):669-676, 2003.

15. FERREIRA, V.A.; MAGALHÃES, R. Obesidade no Brasil: tendências atuais. Revista Portuguesa de Saúde Pública, 24(2): 71-78, 2006.

16. NUNES, M.M.A. et al. Excesso de peso, atividade física e hábitos alimentares entre adolescentes de diferentes classes econômicas em Campina Grande (PB). Rev Assoc Med Bras., 53(2): 130-4, 2007.

17. SARTURI, J.B. et al. Obesidade em adultos: estudo de base populacional num município de pequeno porte no Sul do Brasil em 2005. Ciência \& Saúde Coletiva, 15(1): 105-113, 2010

18. FELIPPE, F.; SANTOS, A.M. Novas demandas profissionais: obesidade em foco. Revista da ADPPUCRS, 5(1): 63$70,2004$.

19. MINISTÉRIO DA SAÚDE. 10 passos para o peso saudável. Disponível em: $<$ http://www.saude.gov.br $>$. Acesso em: 08/Mai/2009.

Endereço para Correspondência:

Elton Bicalho de Souza

elton_bicalho@ig.com.br

Departamento de Nutrição

Centro Universitário de Volta Redonda - UniFOA

Av. Paulo Erlei Alves Abrantes, $n^{\circ} 1325$

Três Poços, Volta Redonda - RJ

CEP 27240-560 